\title{
Study of Modified Epoxy Resin
}

\author{
Shuiyun Ren ${ }^{1}$, Hongjun Huang ${ }^{2}$ \& Zhihong Du $^{3}$ \\ ${ }^{1}$ Mechanical Engineering College, 050003, Shijiazhuang China \\ ${ }^{2}$ Mechanical Engineering College, 050003, Shijiazhuang China \\ ${ }^{3}$ Mechanical Engineering College, 050003, Shijiazhuang China
}

Keywords: Epoxy Resin, toughening, mechanical property.

\begin{abstract}
The paper introduces the basic structure, physical and chemical property as well as the own defects of epoxy(EP), making a summary of main ways of improving EP. By analyzing the advantages and disadvantages of the mechanism and technological approaches which organic silicone, nano particles and polyurethane used in their modification, the paper makes a prospect of the future development trend of EP modification.
\end{abstract}

\section{Introduction}

Through polycondensation reaction of the epoxy compound and polyhydric hydroxy compound, a polymer called epoxy resin is generated, among which the bisphenol A type epoxy resins is the most widespread and classical. The structure shows that EP molecular chain contains more reactive functional groups such as ether bond, epoxy groups and hydroxyl. This structure gives EP excellent chemical properties, for example, high viscosity, good contractability, high stability and good alkali resistance, etc ${ }^{[1]}$. Thus EP is widely used in the furniture, construction, electronics, aerospace, military and other fields. However, because EP contains many benzene or heterocyclic rings, the molecular chain's flexibility bad, cured epoxy resin's crosslink density high and the crosslinked structure hardly deformed, together with high innerstress, leading to that the cured EP is easily brittle, poor impact resistance and easy to peer. Which, to some extent, limit the EP continues to develop and use in many high-tech fields ${ }^{[2]}$.Therefore, in recent years, researchers pay more attention to the toughening study of EP, such as using rubber elastomers, thermoplastic resins, liquid crystal polymers, inorganic nano-fillers, organic silicone, hyperbranched polymer, flexible curing agents, interpenetrating polymer networks and other methods to improve the toughness of $\mathrm{EP}^{[3]}$. In the traditional modification study of EP, rubber elastomers is the most widely used, but the mothed can significantly reduce the strength of EP and the glass transition temperature of resin system, causing inconvenience to the latter application ${ }^{[4]}$.The use of organic silicon, nano-particles and polyurethane will not only toughen EP, but also maintain the shear and tensile strength of the EP, which is applicable potentially. Here the paper will focus on three new methods of EP modification.

\section{Using organic silicone to modified EP}

Organic silicone resins are typically organic - inorganic polymers, based on main molecular chain of Si-O-Si bond, the silicon atom connecting to organic groups. Since the Si-O-Si bond length greater and bond angle large, easy to rotate, the shielding effect of the methyl group on the siliconoxygen chain reduces the interaction between the silicon-oxygen bond, so that the flexible segment of the helical structure can improve the flexibility of the resin[5].Meanwhile, silicone has a high thermal stability, low surface energy, low temperature flexibility, etc., but its cohesive strength low, bonding properties poor. Thus, silicone-modified EP is expected to achieve a complementary effect of their advantages. Using silicone to modified EP is a new developed way recently to toughen EP effectively. Due to the great difference of the solubility parameter between organic silicon and EP, it is essential to solve this problem. Researchers find that introducing functional groups like hydroxyl, carboxyl, amino etc., which can react with the epoxy group to the silicone molecular 
chain, is able to improve the compatibility[6]. Thin Solid Films ,2009,517:4850-4856]. Currently the groups introduced into silicone to enhance compatibility include polar groups of phenyl group, and polyether chain links. They enhance the polarity of the silicone molecules, increasing its compatibility with the epoxy resin[7]. The main ways to modified EP by taking advantage of silicone are as following: 1). By reaction between the isocyanate and the active hydrogen; 2). By radical polymerization; 3). By a condensation reaction; 4). By reaction between reactive groups and epoxy groups; 5). By hydrosilylation of polysilanes containing $\mathrm{Si}-\mathrm{H}$ groups and vinyl resin[8]. Radical polymerization and hydrosilylation reaction is in the most simple forms on siliconemodified EP. Radical polymerization is the use of a modified EP typically functional groups reactive with the acrylic ester monomer and an organic silicon. Xiao Wei bing et al. prepared organosilicon-acrylate-epoxy resin hybrid material by radical solution copolymerization and sol-gel method, in this hybrid material organic phases connect with inorganic phases by chemical bonds, this is a method to modify the resin by radical polymerization. The result showed when mass fractions of tetraethylorthosilicate (TEOS) are $15 \%$ and $25 \%$, the average diameters of inorganic phase $\mathrm{SiO} 2$ particles in the hybrid material are $36 \mathrm{~nm}$ and $45 \mathrm{~nm}$ respectively, and $\mathrm{SiO} 2$ nano particles are homogeneously dispersed within the polymer. When this hybrid material was used in reinforcement of soil for Jinsha archaeological site, results indicated that the hybrid material can effectively fill up cavities within soil and therefore consolidate the soil. Song Xian-gang et al. synthesized the organosilicon-modified water-soluble UV curable epoxy resin with graft copolymerization method by using Epoxy resin E-51 and methyl triethoxysilane as raw materials, and the flexibility, the water solubility and the mechanical properties of the curable resin was effectively improved, this method modified the resin by addition reaction between the polysilane with Si-H radical and the epoxy resin with ethenyl. By measuring, the tensile strength can reach $53.5 \mathrm{MP}$, elongation at break 46.5\%, impact resistance greater than $50 \mathrm{~kg} / \mathrm{cm}$. So far, organ silicone in the epoxy resin modified by organ silicone mostly exist as the 'island' dispersed in the epoxy resin, with small size and homogeneously distribution. Organ silicone greatly changes the epoxy resin in better internal stress, stronger toughness and high temperature resistance. It promotes the epoxy in high performance and multifunction, and provides epoxy resin the potential use and prospect in different areas.

\section{Inorganic Nano material modification}

Nano material has large specific surface area, small particle size, good thermal conductivity, and good dispersion characteristics, especially there are lots of non-stoichiometry atoms on its surface, making it has great possibility to connect with the active groups in the organics by physical or chemical processes. This improves the connection ability between the particles and the surface, and contributes to decrease the polymer bond initiative, and then can ......Meanwhile, because of its excellent thermostability and dimensional stability, etc.advantages, inorganic nano material has been increasingly used in polymer modification. The inorganic nano materials used in the EP modification include $\mathrm{SiO} 2, \mathrm{TiO} 2$ and graphene, etc.

\section{SiO2 modification}

Nano $\mathrm{SiO} 2$ is a kind of white amorphous powder, it has a three-dimensional chain knot structure with a large number of unsaturated bonds and hydroxyl groups bonded to different states. These structures enable the $\mathrm{SiO} 2$ the ability to react tempestuously with the hydroxyl groups and epoxy groups in EP, thus greatly improving the hardness and strength of the material; meanwhile nano $\mathrm{SiO} 2$ dispersing in the resin voids makes a great improvement in polymeric strength, toughness, and ductility.

Wang Chun-qi et al. dispersed the nano-SiO2 into EP, results indicated that the bending strength increased $21.2 \%$ for casting body modified by $1 \mathrm{wt} \% \mathrm{SiO} 2$ and the flexural and tensile strength were increased by $9.7 \%$ and $7.9 \%$, but the interlaminar shear strength was reduced by $10.6 \%$ for its glass fiber reinforced composites. Yang et al. dispersed uniformly the nano-SiO2 into EP .The results indicate that the impact properties, tensile properties, bending properties of EP have been improved significantly, and the EP' other properties have not been influenced. In the two studies, 
the reason why $\mathrm{SiO} 2$ can toughen epoxy resin is that the surface of nano particles which contains plenty of unsaturated bond, with high surface activity, can be used as molecular junction at which point "fracture lines" would be terminated, swerved and deviated, when SiO2 are uniformly dispersed into EP.

\section{TiO2 modification.}

At present, the preparation of TiO2 is usually divided into two categories, the gas phase and liquid phase mothed which has been relatively mature and used in laboratory and factory widely [16]. In recent years, the researchers composite EP with the $\mathrm{TiO} 2$ in order to enhance the toughening polymer. In the modification the active group of $\mathrm{TiO} 2$ which has the surface effect and interface effect combined with the chemical bonds of EP. The modified EP product has been greatly improved in strength, toughness and heat resistance. Zhou hongjun etc. made a mixture of TiO2 and EP ,with MTHPA and DMP-30 (EP mass fraction of 1\%) as accelerant, in the condition of stirring up strongly. Analytically, the introduction of TiO2 reduced the activation energy and promoted the curing reaction of EP, but it didn't change the curing mechanism of EP.

\section{Graphene modification.}

Graphene is a kind of carbonaceous material with carbon atoms tightly packed into a single twodimensional honeycomb lattice structure. Because of its special structure, graphene has excellent rigidity, size stability and toughness etc. Therefore, the introduction of graphene could significantly modify the toughness of epoxy resin. Meanwhile, it can overcome the weakness of the common inorganic materials, such as huge amount of admixture and low modified efficiency [20]. As a kind of inorganic material, oxidized graphene (GO) is made in need of introducing oxygen-containing functional groups such as hydroxyl, carboxyl and epoxy group into graphene. GO combine the group in EP resulting in forming a new bond, so as to improve the material dispersion properties and interfacial effect[21]. In the latest study, the GO in-situ reduction of composite material to the further mechanical strength of the epoxy resin was made by some scholars.

Wang xue bao ect. Made the graphene / epoxy resin composite material through in-situ reduction in which GO had been restored as graphene successfully, its tensile strength and bending strength were maxium increased by about $40.5 \%$ and $9.4 \%$ respectively. Ren xiao meng[23] etc confirmed that graphene has a good toughening effect on epoxy resin by "sea island structure" and "crack nail anchor effect", and GO toughen epoxy resin by silver grain branched mechanism.

Nanoparticles can play a good effect in the EP modification because of its self size effect and huge surface activity. At the same time, nanoparticles reunion phenomenon occur easily in EP,, the reunion chance is higher as its dosage increases, making it difficult for particles to disperse in the epoxy resin, and the concentration of material, and the degradation of material mechanics performance. So, how to nanomaterials better dispersed in matrix material is one of the research at the core of the problem. The dispersion method that had been reported include mechanical stirring, ultrasonic dispersion, dispersion method, etc. the scholars can take different dispersion method, according to different epoxy resin using the environment.

\section{Polyurethane modification.}

Among all the methods of toughening EP, polyurethane modification is the most significant effect. Polyurethane, with both the chain of C-C,C-OC and the activity amide group that provide compatible with EP [24], has good strength, hardness, resistance to flexure, excellent elasticity and organic solvent resistance and other advantages. The mechanism of polyurethane modification of EP is that Polyurethane chain segment run through the EP chain segment, forming interpenetrating polymer network (IPN) or half interpenetrating polymer networks (SIPN) structure, snaring mutually, "forced miscibility" , made the compatibility increased. At the same time because of the polyurethane particles dispersing in the continuous EP, the system toughness increased, the internal stress of EP was dispersed, and its shear strengthen [25].

Kang Si bo, et al[26]. synthesized hydroxyl end group polyurethane pre polymers with polyether diverse and toluene 2, 4 - diisocyanate (TDI) and used it to modify EP. The analysis results show that the formation of IPN, through the polyurethane pre polymers graft modification of EP, can 
significantly improve the hardness of epoxy coating, compression strength, toughness and impact resistance performance, rather than reduce the cohesive strength of the coating. With polyurethane modified EP as he film forming material , Li Wen kai et al. [27] developed a kind coating that has a strong binding power with the based adhesive and an excellent performance for anti-corrosion, when used as anticorrosion primer, it can satisfy the requirement of petrochemical tank walls longterm anti-corrosion. Xi er li et al, synthesized the PU pre polymers/EP (E - 51) graft copolymer using fractional steps. The results showed that when the mass ratio of the PU pre polymers/E - 51is 20:100, the curing system of shear strength ( aluminium / aluminium) up to a maximum of 14.1 MPA, after grafting the condensate is a typical ductile fracture, and graft copolymerization of polyurethane had no effect on the heat resistance of the condensate.

In the above experiments, the successful graft of the polyurethane and the epoxy resin is the direct expression of compatibility between the two. Through observation and analysis, polyurethane has hard segment and soft segment (good soft) which can more effectively modified epoxy resin. In many cases, polyurethane would exist "micro phase separation phenomenon", so if you want to make better use of polyurethane (especially the soft segment) to modify of epoxy resin, researchers should consider the effect of composition of soft segment on the material.

\section{Conclusions}

In the future, we need binary even ternary modification of epoxy resin to further functionalization and high performance. At the same time, there still exists many problems about the epoxy resin modification, for example, a tedious process, high cost, not strong interoperability and so on. Therefore, how to more effectively modify and use epoxy resin in different environment is the future epoxy resin development trend and field.

\section{References}

[1] Yang Hui di. The research progress in chemical modification of epoxy resin. Intelligence development of science and technology and economy, 21, pp.175-177, 2006.

[2] Li Xiaoyu, et al. Synthesisofa hyperebranched polyether epoxy through hone-step proton transfer polymerization and its application as a toughener for epoxy resin. dgeba, 4, pp.493502, 2012.

[3] Liao Bo, Hong-yan Li, Bu-fengzhang, Li Rujian, Franck, The research progress of toughening modification of epoxy resin. Chemical New Materials, 38 (6), pp.28 - 30, 2010.

[4] Qzturk A, Kaynak C, TincerT.Effects of liquid rubber modification on the behavior of epoxy resin[J]. European Polymer Journal, 2001, 37(37), 2253-2363.

[5] Huang W,YaoY,HuangY,et al. Surface modification of epoxy resin by polyetherpolymithylsiloxanes-polyether tri-block copolymers[J]. Polymer,2001,42:1763-1766.

[6] WU L Y,et al. Adhesion enhancement of sol-gel coating on polycarbonate by heated impregnation treatment[J]. Thin Solid Films,2009,517:4850-4856.

[7] Wen Shu feng, The research progress of organic silicon, epoxy polymer [J], Guangdong Chemical Industry, 2009, 4 (10), 104-106.

[8] Zhang dong-yang,et al, The research progress of organic silicone modified resin technology [J], Technical Progress, 2012, 27 (10), 20-23.

[9] XiaoWei Bing, et al.Organic silicon, acrylate, epoxy resin synthesis and performance of the hybrid materials [J], Petrochemical Industry, 2006, 35 (10), 987-993.

[10] Song xian gang et al.Organic silicone modified waterborne epoxy resin research [J], Guangzhou Chemistry, 2013, 20 (3), 5-11.

[11] Sangermano M, Malucelli G, Amerio E et al.Photoplymerization of epoxy coatings containing silicananoparicles [J].Prog Org Coat, 2005,54(2): 134-138.

[12] Liu Ying ling, Hsu C Y, Wang Mei ling, et al. A novel approach of chemical functionalization on nano-scaled silica perticles[J]. Nanotechnology, 2003,14:813-819. 
[13] Azzam R A, Mohamed S K, Tol R, et al. Synthesisi and themo-mechanical characterization of high performance polyurethane elastomers based on heterocyclic and aromatic diamine chain extenders[J]. Polymer Degradation and Stablity, 2007,92(7): 1316-1325.

[14] Wang Chun qi,et al. Nano SiO2 and modified epoxy resin composite material performance study [J], Functional Materials, 2012,22,3045-3048.

[15] Yang Zhi qiang et al. Dispersibility of nano silicon dioxide high voltage insulation research [J] with epoxy resin, insulation materials, 2006 (1), 7- 10.

[16] Liu Yan yan,et al.The preparation of nano titanium dioxide and its application in epoxy resin modified [J], journal of materials science and engineering, 2011, 29 (2), 0316-0320.

[17] Zhou Hong jun, et al. Nano titanium dioxide effect on the cure reaction of epoxy resin [J], thermosetting resin, 2012, 27 (6), 11-14 b.

[18] Tang Long cheng, et al.Graphene/polymer nanocomposites is reviewed [J], tech review, 2013, 31 (27), 71-79.

[19] Wang Deng wu et al. Development on the graphene modification of epoxy resin, [J], China Adhesives, 2013, 22 (7), 42-46.

[20] Yang H, Shan C, Li F, et al. Convenient preparation of tunably loaded chemically converted graphene oxide/epoxy resin nanocomposites from graphene oxide sheets through two-phase extraction[J]. Journal of Materials Chemistry, 2009, 46:8856-8860.

[21] Guo Xiao qin, et al. Graphene preparation of nano/epoxy resin composite materials and dielectric properties research [J], Functional Materials, 2013, 17 (44), 2672-2675.

[22] Wang Xue bao, et al. The preparation of graphene/epoxy resin composite materials and mechanical properties [J], high polymer materials science and engineering, 2013,7,161-165.

[23] Ren Xiao meng, et al. Graphene material on the toughening of epoxy resin and its mechanism research [J], chemical new materials, 2013, 9 (9), 114-116.

[24] THOMAS R, DURIX S, SINTUREL.C, et al. Cure kinetics, morphpology and miscibility of modified dgeba-based epoxy resin effects of a liquid rubber inclusion[J]. Polymer, 2007,48(6): 1696-1712.

[25] KONG J, TANG Y S, ZHANG X J, et al. Synergic effect of acrylate liquid rubber and bisphenol on toughness of epoxy resin[J]. Polymer Bulletin, 2008,60(3): 230-232.

[26] Kang Si bo, et al. The development of the polyurethane modified epoxy anti rolling coating [J], coating industry, lancet, 2013 (7), 63-67.

[27] Li Wen kai et al. The development of the polyurethane modified epoxy anticorrosive coatings [J], corrosion science and protection technology, 2013, 25 (1), 53-57. 